\title{
Air/water interfacial assembled rubbery semiconducting nanofilm for fully rubbery integrated electronics
}

\author{
Ying-Shi Guan ${ }^{1}$, Anish Thukral ${ }^{1}$, Shun Zhang ${ }^{2}$, Kyoseung Sim ${ }^{3,4}$, Xu Wang $^{3}$, Yongcao Zhang ${ }^{3}$, \\ Faheem Ershad ${ }^{5}$, Zhoulyu Rao ${ }^{3}$, Fengjiao Pan ${ }^{1}$, Peng Wang ${ }^{3}$, Jianliang Xiao ${ }^{2}$, Cunjiang Yu $\mathbf{u}^{1,3,5,6,7 *}$
}

A rubber-like stretchable semiconductor with high carrier mobility is the most important yet challenging material for constructing rubbery electronics and circuits with mechanical softness and stretchability at both microscopic (material) and macroscopic (structural) levels for many emerging applications. However, the development of such a rubbery semiconductor is still nascent. Here, we report the scalable manufacturing of high-performance stretchable semiconducting nanofilms and the development of fully rubbery transistors, integrated electronics, and functional devices. The rubbery semiconductor is assembled into a freestanding binary-phased composite nanofilm based on the air/water interfacial assembly method. Fully rubbery transistors and integrated electronics, including logic gates and an active matrix, were developed, and their electrical performances were retained even when stretched by $50 \%$. An elastic smart skin for multiplexed spatiotemporal mapping of physical pressing and a medical robotic hand equipped with rubbery multifunctional electronic skin was developed to show the applications of fully rubbery-integrated functional devices.

\section{INTRODUCTION}

Electronics and circuits fully composed of rubbery electronic materials with mechanical softness and stretchability at both microscopic (material) and macroscopic (structural) levels are critical for many technically challenging yet important applications such as electronicsorgan symbiosis (1), humanoids $(2,3)$, and wearable (4-7) and implantable bioelectronics (8-11). Although significant advancement on stretchable electronics has been achieved based on nonstretchable materials, such as $\mathrm{Si}, \mathrm{GaAs}$ with structural design strategies enabling mechanical stretchability, these materials are intrinsically rigid at the microscopic (material) level $(12,13)$. To create fully rubbery electronics and circuits, the rubbery semiconductor is an indispensable material (14-20). Meanwhile, it is critically important that the rubbery semiconductor has high carrier mobility and can be scalably manufactured to achieve high-performance integrated electronics and systems to meet the aforementioned applications $(21,22)$. Although there have been a few recent reports on creating stretchable semiconductors (23-27), the material development is still nascent. The reported stretchable semiconductors are primarily based on solution spin coating, solution shearing, or drop casting of semiconducting composites $(17,23,24)$. The devices fabricated using those approaches either have relatively low carrier mobility or lack device uniformity for integrated electronics. Therefore, to fully realize the practical usage of rubbery electronics and circuits, a technology

\footnotetext{
'Department of Mechanical Engineering, University of Houston, Houston, TX 77204, USA. ${ }^{2}$ Department of Mechanical Engineering, University of Colorado, Boulder, CO 80303, USA. ${ }^{3}$ Materials Science and Engineering Program, University of Houston, Houston, TX 77204, USA. ${ }^{4}$ Department of Chemistry, Ulsan National Institute of Science and Technology (UNIST), Ulsan 44919, Republic of Korea. ${ }^{5}$ Department of Biomedical Engineering, University of Houston, Houston, TX 77204, USA. ${ }^{6}$ Department of Electrical and Computer Engineering, University of Houston, Houston, TX 77204, USA. ${ }^{7}$ Texas Center for Superconductivity, University of Houston, Houston, TX 77204, USA.

*Corresponding author. Email: cyu15@uh.edu
}

gap in scalable manufacturing of high mobility, stretchable semiconductors must be bridged.

Here, we report the facile and scalable manufacturing of highperformance stretchable semiconducting nanofilms and the fully rubbery transistors, integrated electronics, and functional devices. Specifically, the rubbery semiconducting nanofilm is scalably manufactured into a freestanding format based on air/water interfacial assembly methods, which particularly leads to favorable molecular packing of poly(3-hexylthiophene) (P3HT) molecules and the resulting high-field effect carrier mobility. The binary-phased rubbery composite semiconducting nanofilm advantageously offers mechanical stretchability. The yielded composite nanofilm has distinct advantages: (i) high charge carrier mobility $\left(8.57 \mathrm{~cm}^{2} \mathrm{~V}^{-1} \mathrm{~s}^{-1}\right)$; (ii) excellent mechanical stretchability by $50 \%$ without significant decay in carrier mobility; and (iii) simple, scalable, repeatable, and low-cost manufacturing. Rubbery transistors and their arrays made fully out of rubbery conductor, semiconductor, and dielectric have been developed. The rubbery transistors can be stretched up to $50 \%$ with a reasonable decrease in their mobility. In addition, fully rubbery logic gates have been realized and their logical functions were maintained even under $50 \%$ applied strain. Furthermore, an elastic smart skin with a $5 \times 5$ rubbery transistor array-based active matrix with multiplexed spatiotemporal mapping capability was developed. Last, a multifunctional rubbery electronic skin (E-skin) was developed and implemented on a robotic hand to illustrate its function as an effective medical diagnostic device. The air/water interfacial assembled semiconducting nanofilm and rubbery electronic devices described here suggest a feasible pathway toward rubbery electronics and integrated systems and their broad emerging applications.

\section{RESULTS}

\section{Freestanding rubbery semiconducting nanofilm}

The air/water interfacial assembly of freestanding nanofilms under ambient conditions is schematically illustrated in Fig. 1A. We first 

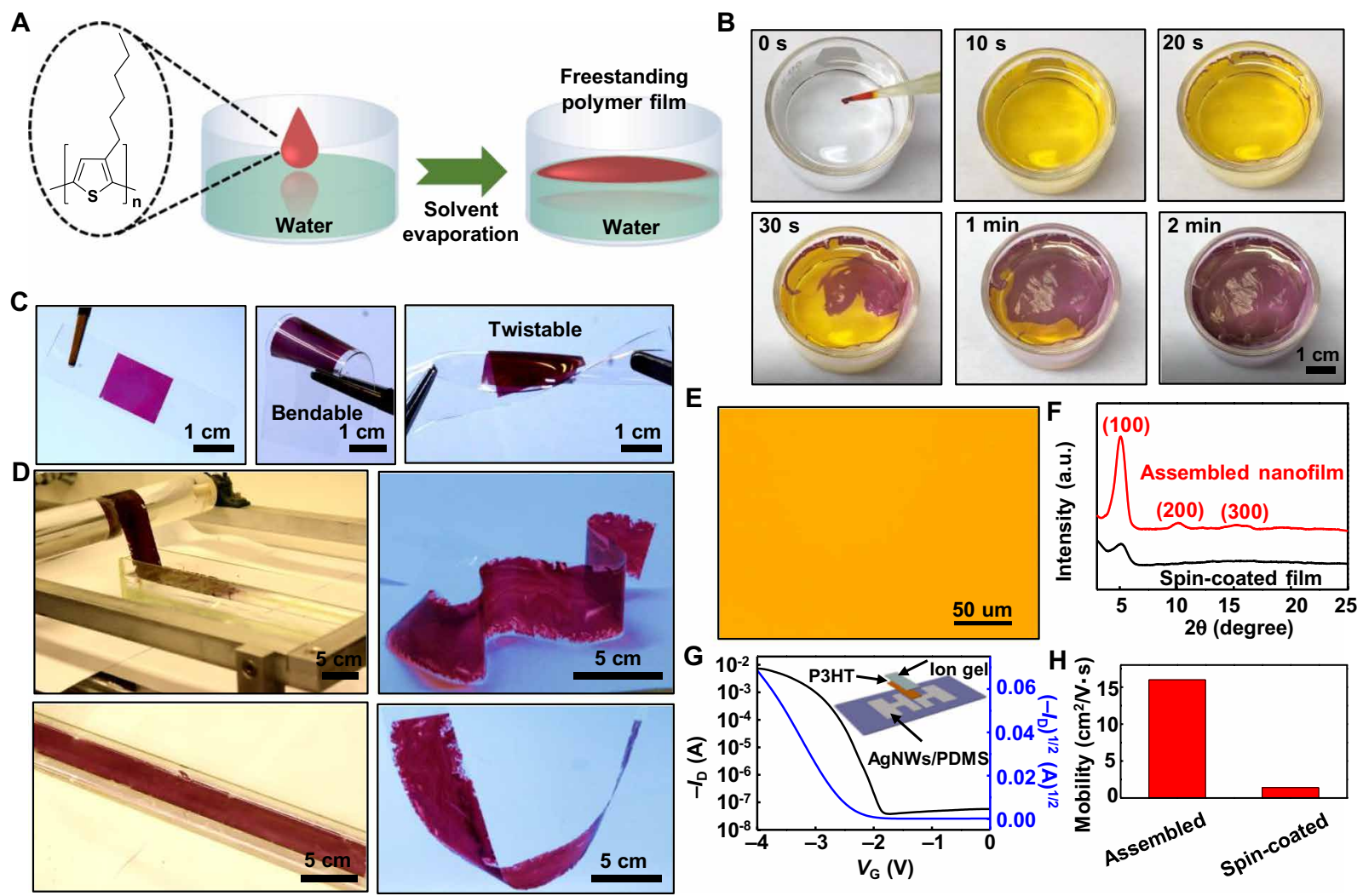

Fig. 1. The fabrication and characterization of the assembled freestanding pristine $\mathrm{P} 3 \mathrm{HT}$ nanofilm. (A) Chemical structure of $\mathrm{P} 3 \mathrm{HT}$ and schematic illustration of the formation process of the polymer nanofilm on the water surface. (B) Photographs of the pristine P3HT nanofilm formed on the water surface. (C) Assembled freestanding pristine P3HT nanofilm deposited on a PDMS substrate, in bent and twisted states. (D) Large-area fabrication demonstrated by a roll-to-roll process. (E) Optical image of the pristine P3HT film deposited on a Si substrate. (F) XRD pattern of the assembled freestanding pristine P3HT nanofilm and spin-coated film. a.u., arbitrary units. (G) Representative transfer curve of the assembled freestanding pristine $\mathrm{P} 3 \mathrm{HT}$ nanofilm-based transistor. (H) Mobility values of the assembled freestanding pristine $\mathrm{P} 3 \mathrm{HT}$ nanofilm and spin-coated film. Photo credit: Ying-Shi Guan, University of Houston.

created a pristine $\mathrm{P} 3 \mathrm{HT}$ nanofilm based on such an assembly method. By dissolving P3HT in toluene, the polymer solution was formed. Because the toluene is immiscible with water, as soon as the polymer solution is gently dropped onto water, it spreads out on the water surface rapidly, which is known as the Marangoni effect $(28,29)$. A nanofilm is therefore formed as the solvent evaporates and it floats on the wafer surface. Figure $1 \mathrm{~B}$ shows the sequential images of the assembly processes for the pristine $\mathrm{P} 3 \mathrm{HT}$ nanofilm, which formed within about $2 \mathrm{~min}$. During the toluene evaporation process, $\mathrm{P} 3 \mathrm{HT}$ molecules at the air/water interface could effectively self-assemble into well-ordered structures through intermolecular interactions (30) such as $\pi-\pi$ stacking (31-33) and hydrophobic interaction (34). Because of the hydrophobic nature of the polymer, the polymer chains tend to pack densely to minimize the contact with water during the solvent evaporation, which leads to a strong $\pi-\pi$ stacking. The thickness of the yielded film is well controlled based on the amount of the polymer solution applied. The nanofilm can remain freestanding and structurally stable for a relatively long period of time (e.g., 6 months) without additional treatment. The freestanding nanofilm can be picked up carefully in water (fig. S1) and harvested onto various substrates (35), such as a glass slide and a piece of curved plastic (fig. S2). Figure 1C shows the photos of the nanofilm transferred onto a thin polydimethylsiloxane (PDMS) substrate, which was also bent and twisted. The nanofilm assembly and sub- sequently harvested onto a target substrate can be implemented in a scalable roll-to-roll manner, as shown in Fig. 1D. The nanofilm was transferred onto a 5 -cm-wide polyethylene terephthalate film through rolling at the speed of $5 \mathrm{~mm} \mathrm{~s}^{-1}$. Figure $1 \mathrm{E}$ shows the optical microscope image of a nanofilm on a Si wafer. The nanofilm is measured to be $60 \mathrm{~nm}$ thick (fig. S3) and is continuous and uniform across the whole wafer. X-ray diffraction (XRD) spectroscopy was used to gain insight into the molecular packing within the nanofilm. As shown in Fig. 1F, the assembled pristine $\mathrm{P} 3 \mathrm{HT}$ nanofilm (in red) exhibits three distinct multiorder [ $(h 00), h=1,2,3]$ diffraction peaks at $5.2^{\circ}, 10.5^{\circ}$, and $15.7^{\circ}$, while the spin-coated film (in black) showed only one broad and weak diffraction peak (100). The results indicate that a highly ordered packing structure exists within the pristine P3HT nanofilm, as shown in fig. S4. Such a packing structure is similar to that existing in the crystalline P3HT nanofibers (36).

The carrier mobility of the pristine P3HT nanofilm was investigated based on transistor devices. Specifically, a conductive composite of $\mathrm{Au}$ nanoparticles with conformally coated silver nanowires (AuNP-AgNW) dispersed within PDMS elastomer (AuNPs-AgNWs/ PDMS) was used as source and drain electrodes, and ion gel was used as a dielectric layer. These materials were chosen because (i) they are mechanically stretchable and rubber-like as reported elsewhere (24) and (ii) the same materials were used in rubbery transistors. To fabricate the transistors, a pristine $\mathrm{P} 3 \mathrm{HT}$ nanofilm 
was transferred onto a PDMS substrate with prefabricated AuNPsAgNWs/PDMS stretchable electrodes. Then, a freestanding ion gel sheet was laminated onto the surface of the pristine P3HT nanofilm to form the gate dielectric. The detailed electrode preparation and device fabrication are presented in Materials and Methods and have been reported elsewhere (26). Figure $1 \mathrm{G}$ shows the transfer curve of the transistor with $p$-type characteristics, where the gate voltage $V_{\mathrm{G}}$ was swept from 0 to $-4 \mathrm{~V}$ and the drain voltage $\left(V_{\mathrm{D}}\right)$ was fixed at $-1 \mathrm{~V}$, with the current on/off ratio of $2 \times 10^{5}$. The gate current of the transistor is shown in fig. S5, where the gate leakage current is much smaller than the on current of the transistor. The output curve of the transistor is shown in fig. S6. The average field effect mobility is calculated to be $16.7 \mathrm{~cm}^{2} \mathrm{~V}^{-1} \mathrm{~s}^{-1}$. The detailed calculations are presented in the Supplementary Materials. For comparison, transistors with the spin-coated P3HT film and the same device configuration were fabricated and evaluated. The transfer curve of the spin-coated film-based transistor is shown in fig. S7. The calculated average field effect mobility is $1.14 \mathrm{~cm}^{2} \mathrm{~V}^{-1} \mathrm{~s}^{-1}$. As shown in Fig. $1 \mathrm{H}$, the assembled pristine P3HT nanofilm has a much higher carrier mobility than that of the spin-coated P3HT film because of the highly ordered crystalline packing structure within the assembled nanofilm. The charge transport along the $\pi$-stacking direction is sensitive to the packing structure because the electron coupling is dictated by the thiophene ring alignment, which can form conductive networks to facilitate the charge carrier transport. In the spin-coated P3HT film, there is no long-range ordered packing structure to form conductive networks. The tunneling resistance between the neighboring grain boundaries would impede the electron coupling and eliminate charge transfer. Therefore, the assembled nanofilm exhibited much higher charge carrier mobility than that of the spin-coated film. That pristine $\mathrm{P} 3 \mathrm{HT}$ nanofilm that has higher mobility agrees well with the XRD results. The pristine P3HT nanofilm is not stretchable (fig. S8A). As the nanofilm is stretched, cracks immediately appear. Figure S8B shows the transfer curves of the transistor based on the pristine P3HT nanofilm upon uniaxially stretched by $8 \%$.

To impart mechanical stretchability into the nanofilm, a polystyreneblock-poly(ethylene-ran-butylene)-block-polystyrene (SEBS) elastomer was blended into the P3HT-toluene polymer solution to form a composite. Generally, a composite film with SEBS as the polymer matrix inherits the mechanical stretchability from SEBS. SEBS-based semiconducting composite nanofilms were manufactured based on the aforementioned air/water interfacial assembly process. The detailed preparation of the polymer solution and composite nanofilms is described in Materials and Methods. Composite nanofilms with different weight percentages of P3HT were prepared, and their structures and mechanical stretchability were investigated. Figure 2 (A to E) shows a set of optical images of the composite nanofilms before and after stretching (50\%). In the composite nanofilms with high percentage [such as 85 and 75 weight \% (wt \%)] of P3HT (i.e., lower percentage of SEBS), as shown by the bottom images of Fig. 2 (A and B), visible cracks present as the composite nanofilms are stretched. In contrast, in the composite nanofilms with relatively lower percentage (such as 65,50 , and 35 wt \%) of P3HT (i.e., higher percentage of SEBS), as shown by the bottom images of Fig. 2 (C to E), no crack was observed. The mechanical stretchability is significantly enhanced after the introduction of a certain amount of SEBS elastomer into the assembled P3HT nanofilm.

The carrier mobilities of the semiconducting composite nanofilms with different percentages of $\mathrm{P} 3 \mathrm{HT}$ were also investigated. The materials preparation and device fabrication follow the aforementioned procedures for the transistors with the pristine P3HT nanofilm, as detailed in Materials and Methods. Figure 2F shows the transfer curves of the devices with composite nanofilms with different wt \% of P3HT. The calculated carrier mobilities of the composite nanofilms are summarized in Fig. 2G. The detailed statistical results are shown in fig. S9. The mobility increases as the wt \% P3HT increases. Specifically, the mobilities are $12.37,10.58$, and $8.54 \mathrm{~cm}^{2} \mathrm{~V}^{-1} \mathrm{~s}^{-1}$ for the nanofilm with 85,75 , and 65 wt \% P3HT, respectively. The mobility drastically decreases to 1.56 and $1.01 \mathrm{~cm}^{2} \mathrm{~V}^{-1} \mathrm{~s}^{-1}$ for the nanofilm with 50 and 35 wt \% P3HT, respectively. Because only composite nanofilms with relatively lower percentage (i.e., 65, 50, and 35 wt \%) of $\mathrm{P} 3 \mathrm{HT}$ are mechanically stretchable, we therefore chose the composite nanofilm with $65 \mathrm{wt} \% \mathrm{P} 3 \mathrm{HT}$ as the rubbery semiconductor to further investigate its microstructures and used it for rubbery electronic construction. The XRD pattern of the composite nanofilm with $65 \mathrm{wt} \% \mathrm{P} 3 \mathrm{HT}$ is shown in fig. S10, which exhibits two strong diffraction peaks of crystallized P3HT. The results indicate that highly ordered P3HT molecular packing structures also exist in the composite nanofilm with 65 wt \% P3HT, which is also verified by the relatively high carrier mobility $\left(8.54 \mathrm{~cm}^{2} \mathrm{~V}^{-1} \mathrm{~s}^{-1}\right)$.

The rubbery semiconductor (i.e., composite nanofilm with $65 \mathrm{wt} \%$ P3HT) exhibits binary phases, as shown by the optical microscope images in Fig. 2H. The thickness of the composite nanofilm is about $80 \mathrm{~nm}$ (fig. S10). One phase is a continuous mesh domain throughout the nanofilm; the other is an isolated island-like domain dispersed in the continuous phase. In addition, compared to Fig. 2I, the top and bottom surface topographic structures are almost identical, which suggests that the composite nanofilm has only in-plane but no vertical phase segregation. To determine the composition of the two phases, the composite nanofilm was immersed in cyclohexane to selectively dissolve the SEBS while maintaining P3HT. After $\sim 40$ hours, the isolated island-like domain phase was mostly removed, while the continuous mesh domain phase was mostly retained (Fig. 2J). Further atomic force microscopic (AFM) investigation of the nanoscale morphology of the continuous phase, as shown in Fig. 2K, suggests that the continuous phase contains SEBS and was removed by cyclohexane. These results indicate that the continuous mesh domain phase is P3HT rich and the island-like domain phase is SEBS rich, as also illustrated by the AFM image in Fig. 2L. The associated phase separation in the composite nanofilm can be explained as follows. On one hand, the significantly lower surface energy of both the P3HT-rich (10.57 mN/m for pure P3HT) and SEBS-rich $(14.81 \mathrm{mN} / \mathrm{m}$ for pure SEBS) phases (23) compared with the water $(72.5 \mathrm{mN} / \mathrm{m}$, $20^{\circ} \mathrm{C}$ ) phase (37) leads to no vertical phase separation. On the other hand, the interfacial tension between the P3HT and SEBS phases leads to in-plane separation during the toluene solvent evaporation. In addition, because SEBS has a higher solubility than P3HT in toluene (25), the P3HT tends to first precipitate during the toluene evaporation process. Consequently, the SEBS-rich phase tends to form isolated domains dispersed in the P3HT-rich phase due to the surface tension effect. Such mesh-structured binary phases give rise to the large mechanical stretchability. The rubbery semiconducting nanofilm transferred onto a PDMS substrate can be reversibly stretched by $50 \%$ and released as shown in Fig. $2 \mathrm{M}$.

To better understand its mechanical behavior, a three-dimensional (3D) finite element modeling (FEM) simulation was established to simulate the stretching behavior of the rubbery semiconducting nanofilm on a PDMS substrate in Abaqus. The strain distribution in 
A
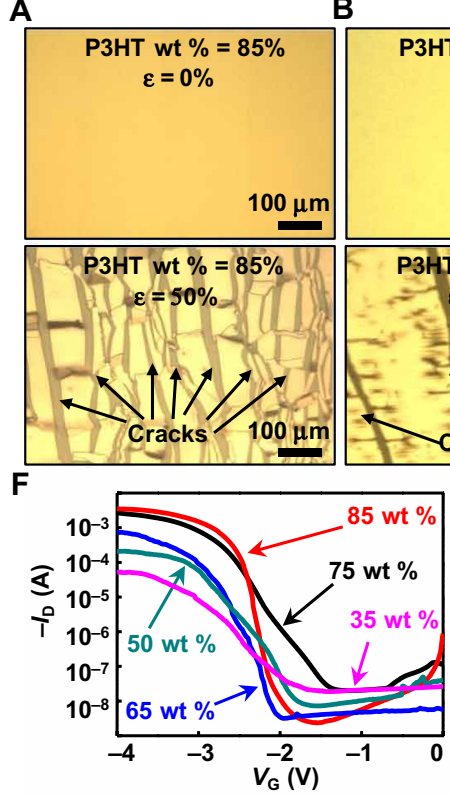

$\mathbf{J}$

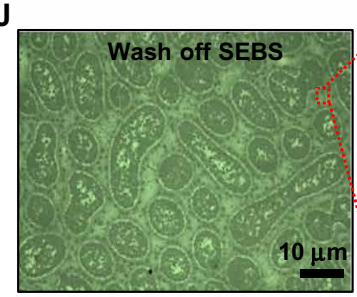

B C

C
E
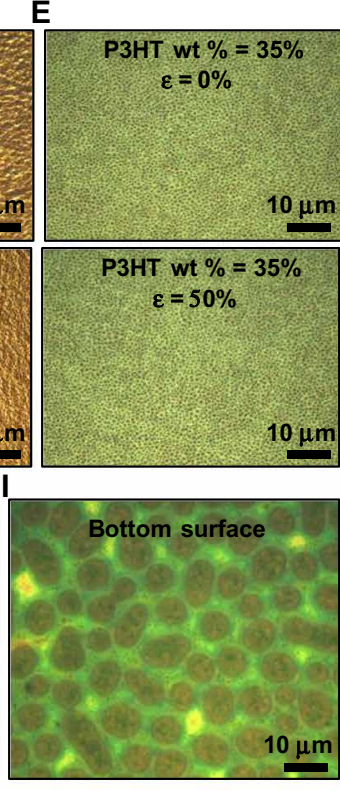

M

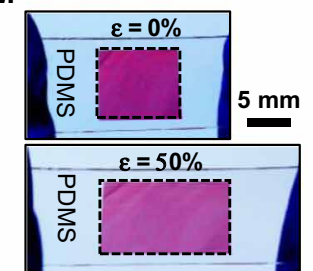

Fig. 2. The assembled freestanding composite nanofilm with different percentages of P3HT. (A to E) Optical images of the composite nanofilm with different percentages of P3HT at 0\% (top frames) and 50\% strain (bottom frames). (F) Representative transfer curve of the composite nanofilm with different percentages of P3HT. (G) Mobility values of the composite nanofilm-based transistor with different percentages of P3HT. (H and I) Optical images of the top and bottom surfaces of the $65 \mathrm{wt}$ \% P3HT composite nanofilm located in the same position, respectively. (J) Optical image of the $65 \mathrm{wt} \%$ P3HT composite nanofilm after washing off the SEBS part. (K) AFM image of the P3HT-rich phase. (L) AFM image of the $65 \mathrm{wt} \%$ P3HT composite nanofilm marked by the composition of the two separated phases. (M) Photograph of the $65 \mathrm{wt} \% \mathrm{P} 3 \mathrm{HT}$ composite nanofilm deposited on a rubbery substrate, showing its high stretchability.

the rubbery semiconducting nanofilm with $50 \%$ strain is shown in fig. S11. The strain distributed in the majority of the SEBS-rich phase is higher than 50\%, while the strain distributed in the P3HTrich phase has a much lower magnitude. In other words, the SEBSrich phase bears the most strain when the rubbery composite nanofilm is stretched. On the other hand, the P3HT-rich phase behaves like a mesh structure at nanoscale (Fig. 2K), which enables the P3HT-rich phase to withstand a relatively larger strain than that for pristine P3HT films. These experiment and simulation results collectively provide the rationale that the rubbery semiconducting composite nanofilm holds excellent mechanical stretchability. Although studies have reported the assembly of semiconductor film on water based on a two-material precursor (organic semiconductor and solvent) system (31-33), here, this work is a three-material precursor (organic semiconductor, SEBS rubber, and solvent) system to introduce unique phase separation to generate a mesh-like microstructure with large mechanical stretchability while retaining high mobility.

\section{Rubbery transistors}

Rubbery transistors based on rubbery semiconducting composite nanofilm were fabricated. Their electrical characteristics under different levels of mechanical strain were measured. The rubbery transistors retain their functions even under $50 \%$ applied mechanical strain. Figure 3 ( $A$ and $B$ ) shows the transfer curves of the rubbery transistor under $0,10,30,50$, and $0 \%$ (released) uniaxial strain along and perpendicular to the channel length direction, respectively. When the transistor was stretched perpendicular to the channel length direction, the on current of the transistor shows a monotonic decrease from $1.81 \mathrm{~mA}$ at $0 \%$ to $1.67 \mathrm{~mA}$ at $10 \%$, to $1.10 \mathrm{~mA}$ at $30 \%$, and to $0.90 \mathrm{~mA}$ at $50 \%$ strain. The current went back to $1.34 \mathrm{~mA}$ when the stretching was released. The off current of the transistor shows a slight change from $9.88 \mathrm{nA}$ at $0 \%$ to $9.66 \mathrm{nA}$ at $10 \%$, to $10.07 \mathrm{nA}$ at $30 \%$, and to $18.57 \mathrm{nA}$ at $50 \%$ strain. The current went back to $15.8 \mathrm{nA}$ when the stretching was released. The corresponding on/off ratio decreased from $1.83 \times 10^{5}$ at $0 \%$ to $1.72 \times 10^{5}$ at $10 \%$, to $1.09 \times 10^{5}$ at $30 \%$, to $0.48 \times 10^{5}$ at $50 \%$ strain, and to $0.85 \times 10^{5}$ at $0 \%$ strain, upon releasing the stretching. The threshold voltage remained at $\sim 2.55 \mathrm{~V}$ when stretched at different levels. The calculated mobilities under different levels of mechanical strain are presented in Fig. 3C. The average mobility experienced a slight decrease (5.8\%) from $8.6 \mathrm{~cm}^{2} \mathrm{~V}^{-1} \mathrm{~s}^{-1}$ to $8.1 \mathrm{~cm}^{2} \mathrm{~V}^{-1} \mathrm{~s}^{-1}$ when the device was stretched by $30 \%$ strain along the channel length direction, and a moderate decrease (20.9\%) to $6.8 \mathrm{~cm}^{2} \mathrm{~V}^{-1} \mathrm{~s}^{-1}$ when stretched by $50 \%$. After the mechanical stretching was released, the average mobility 
A

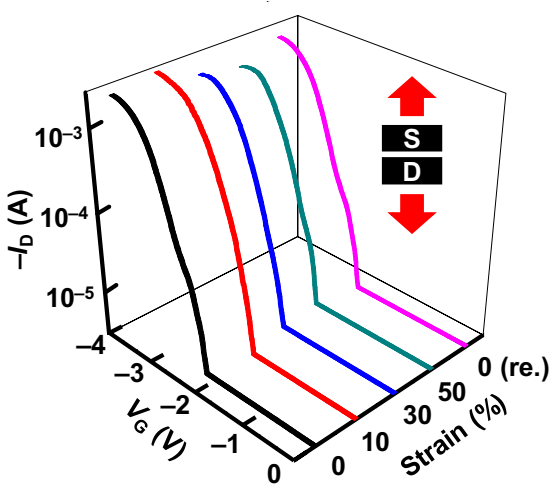

C

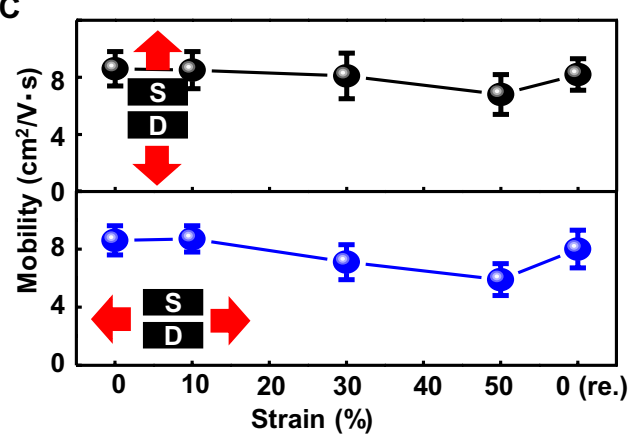

E

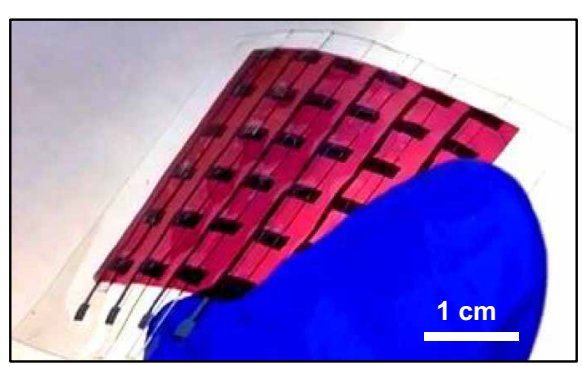

B

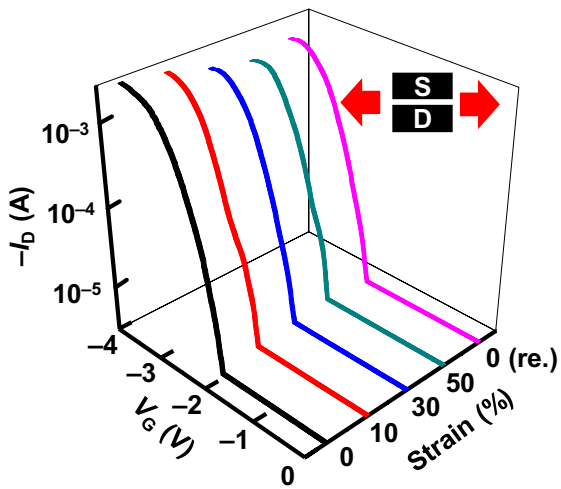

D

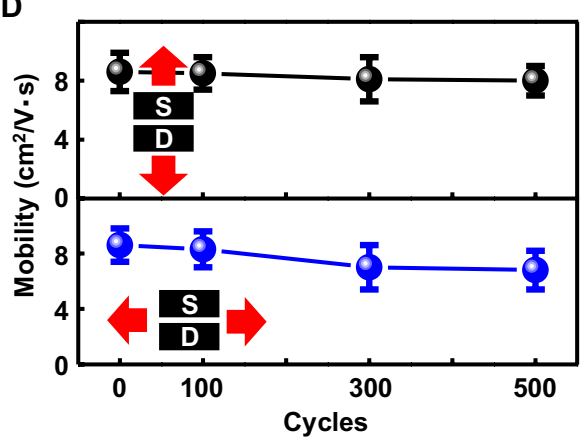

$\mathbf{F}$

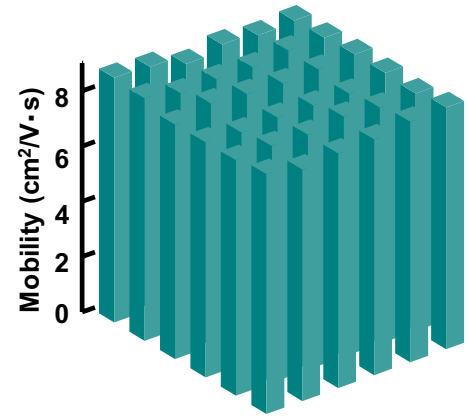

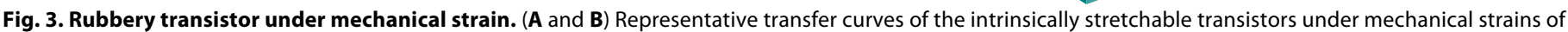

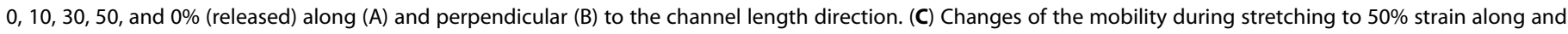

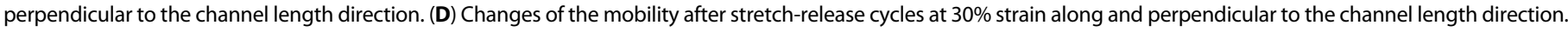
(E) Photograph of the transistor array. (F) Mobility distribution in the transistor array. Photo credit: Ying-Shi Guan, University of Houston.

was mostly recovered $\left(8.2 \mathrm{~cm}^{2} \mathrm{~V}^{-1} \mathrm{~s}^{-1}\right)$. Similarly, when the device was stretched along the direction perpendicular to the channel length, the average mobility slightly decreased to $7.1 \mathrm{~cm}^{2} \mathrm{~V}^{-1} \mathrm{~s}^{-1}$ at $30 \%$ strain and to $5.9 \mathrm{~cm}^{2} \mathrm{~V}^{-1} \mathrm{~s}^{-1}$ at $50 \%$ strain. The average mobility was recovered to $8.0 \mathrm{~cm}^{2} \mathrm{~V}^{-1} \mathrm{~s}^{-1}$ when the stretching was fully released. These results show that the rubbery transistor can function well under large mechanical strain. In addition, to evaluate its reliability under cyclic deformation, the transistor was repeatedly stretched and released for 500 times by $30 \%$ (Fig. 3D). The average mobility slightly decreased to $8.0 \mathrm{~cm}^{2} \mathrm{~V}^{-1} \mathrm{~s}^{-1}$ and to $7.0 \mathrm{~cm}^{2} \mathrm{~V}^{-1} \mathrm{~s}^{-1}$ after 500 cycles of stretching and releasing along and perpendicular to the channel length direction, respectively. Figure S12 shows the cyclic stretching and releasing test at $30 \%$ strain for 500 cycles. Table S1 shows the comparison of our fully stretchable transistors with reported fully rubbery stretchable transistors.

We further constructed an array of rubbery transistors to illustrate the device uniformity, which is critical for integrated electronics. The photograph of a $6 \times 6$ transistor array is shown in Fig. 3E. The schematic illustration of the array is shown in fig. S13A, and the detailed fabrication processes are described in Materials and Methods. The transistors in the array have a high yield of $100 \%$, as can be seen from the array's mobility map shown in Fig. 3F. As also illustrated in fig. $\mathrm{S} 13 \mathrm{~B}$, the highest and average mobilities are 8.85 and $8.57 \mathrm{~cm}^{2} \mathrm{~V}^{-1} \mathrm{~s}^{-1}$, respectively. The array also shows a fairly uniform on/off current ratio with an average value of $3.25 \times 10^{5}$ (fig. S14). In addition, the hysteresis loop of the rubbery transistor is shown in fig. S15, which shows a slight and fairly reasonable hysteresis for ion gel-based transistors (38). Figure S16 shows the initial transfer curves measured in ambient air and after 7 and 15 days of storage in air. The overall current-voltage curves did not change much, but the threshold voltage shifted slightly to positive value and the on/off ratio decreased slightly after 7 and 15 days.

\section{Rubbery logic gates}

Logic gates are basic functional units in integrated electronics and circuits (39). By structuring the logic gates in rubbery formats, they 
can be used for many important yet technically challenging applications (40). Fully rubbery logic gates were developed based on the rubbery transistors, using the processes described previously. Figure 4 (A to $\mathrm{C}$ ) shows the exploded view and circuit diagrams of the rubbery inverter, NAND, and NOR logic gates, respectively. Their corresponding photographs are shown in Fig. 4D. Detailed fabrication processes of the logic gates are described in Materials and Methods. The rubbery inverter was designed to be a zero- $V_{\mathrm{GS}}$ load unipolar inverter based on two $p$-type rubbery transistors (Fig. 4A). Specifically, the driver and load transistors have the same channel length and a channel width ratio of 1:4. The voltage transfer characteristics (VTCs) of the rubbery inverter are plotted in Fig. 4E, which was obtained by measuring the output voltage changes while sweeping the input voltage from -2 to $0 \mathrm{~V}$ with a fixed $V_{\mathrm{dd}}$ of $1 \mathrm{~V}$. The calculated voltage gain of the inverter was $\sim 15$, as shown in Fig. $4 \mathrm{E}$. The rubbery inverter exhibits slight hysteresis, as shown in fig. S17. Figure 4 (F and $\mathrm{G}$ ) shows the VTCs of the rubbery inverter under 0 , $10,30,50$, and $0 \%$ (released) uniaxial strain along and perpendicular to the channel length direction, respectively. Although the VTCs slightly shift, the inverters under different levels of strain all operate normally with logic output states of 1 and 0 under logic input states of 0 and 1 , respectively. Furthermore, the rubbery inverter was cyclically stretched and released by $30 \%$ for 100 cycles. The measured VTC curves (Fig. 4, H and I) show that the device functioned reliability.

Rubbery NAND and NOR gates were designed with a structure of two-driver and one-load transistors. For the NAND gates, the two parallel driver rubbery transistors were connected serially to the load transistor. The channel length was the same, and the channel width ratio between the driver and the load transistor was 1:4. For the NOR gates, the two-driver transistors and one-load transistor were serially connected together. The channel width ratio between the driver and the load transistor was 1:3. The logic gate functions were characterized by sweeping the input voltage from -2 to $0 \mathrm{~V}$ under a fixed $V_{\mathrm{dd}}$ of $1 \mathrm{~V}$. The input voltages of -2 and $0 \mathrm{~V}$ represent input logic states 0 and 1 for $V_{\mathrm{in}, \mathrm{A}}$ and $V_{\mathrm{in}, \mathrm{B}}$, respectively. The measured $V_{\text {out }}$ of the NAND gate shows logic state 0 only when both inputs $\left(V_{\mathrm{in}, \mathrm{A}}\right.$ and $\left.V_{\mathrm{in}, \mathrm{B}}\right)$ are logic state 1 (Fig. $4 \mathrm{~J}$ and fig. S18). Even under $50 \%$ strain along and perpendicular to the channel length direction, the NAND gate retained correct output logic (Fig. 4, K and L), respectively. The measured $V_{\text {out }}$ of NOR gate shows logic state 1 only when both inputs $\left(V_{\mathrm{in}, \mathrm{A}}\right.$ and $\left.V_{\mathrm{in}, \mathrm{B}}\right)$ are logic state 0 (Fig. $4 \mathrm{M}$ and fig. S19). The NAND gate also retained correct output logic (Fig. $4, \mathrm{~N}$ and $\mathrm{O}$ ) under $50 \%$ strain along and perpendicular to the channel length direction, respectively. Both NAND and NOR gates exhibited well-defined high and low output logic levels and functioned
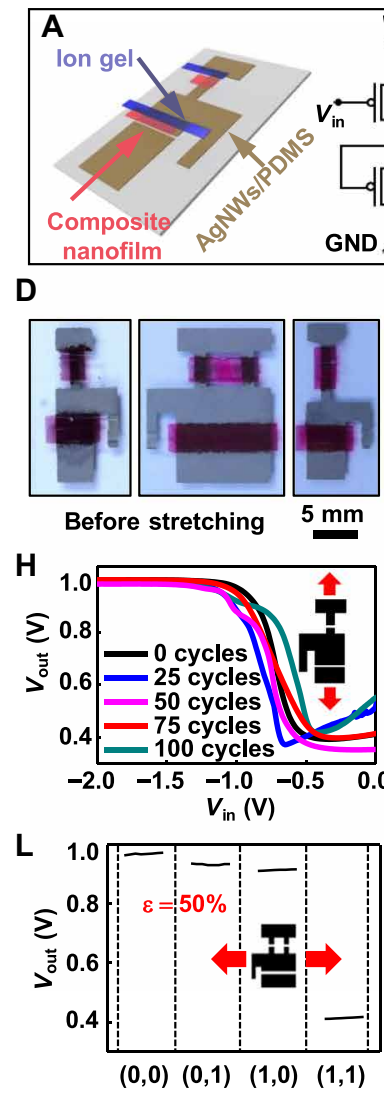
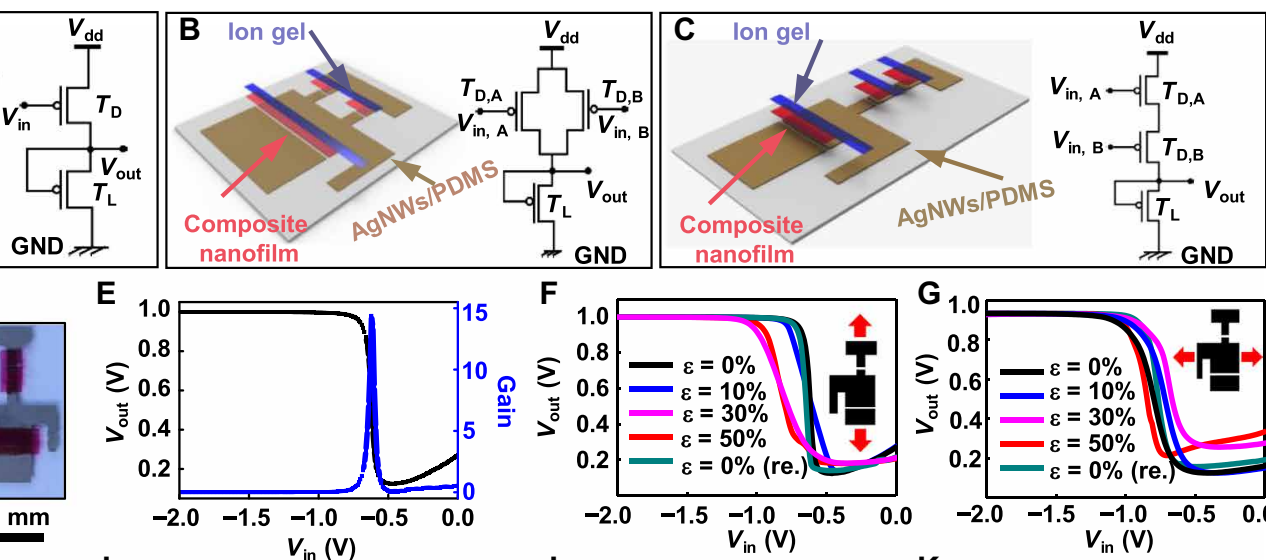

$F$
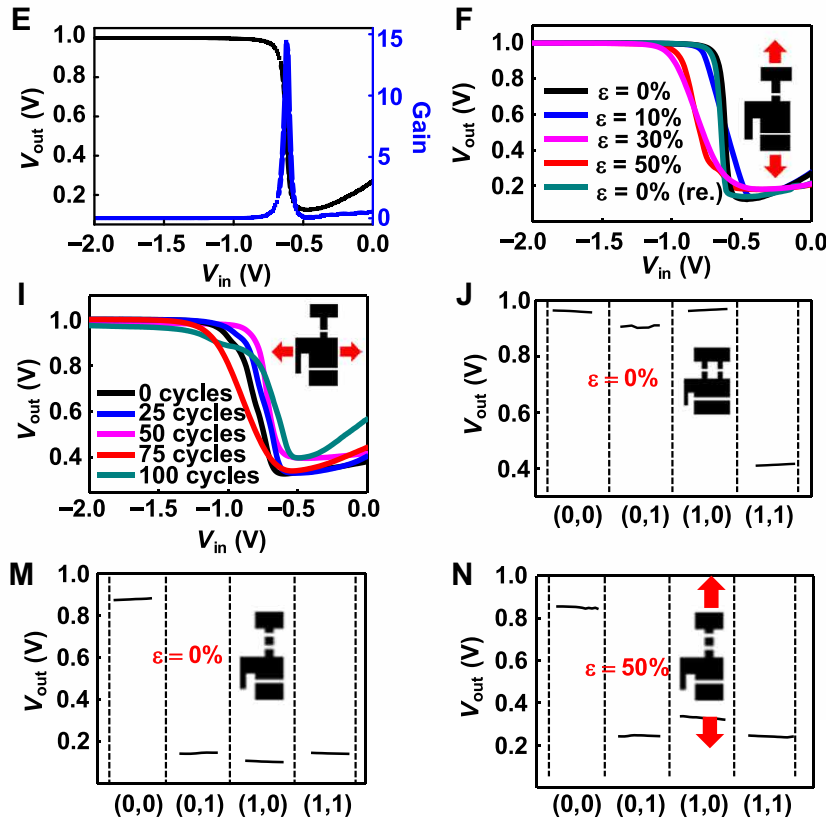

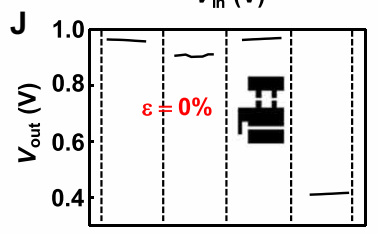

$(0,0)(0,1)(1,0)(1,1)$

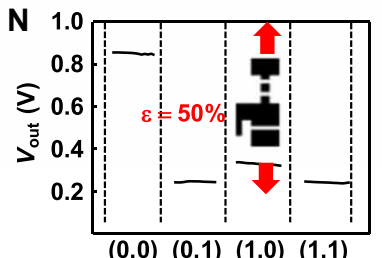

$(0,0)(0,1)(1,0)(1,1)$
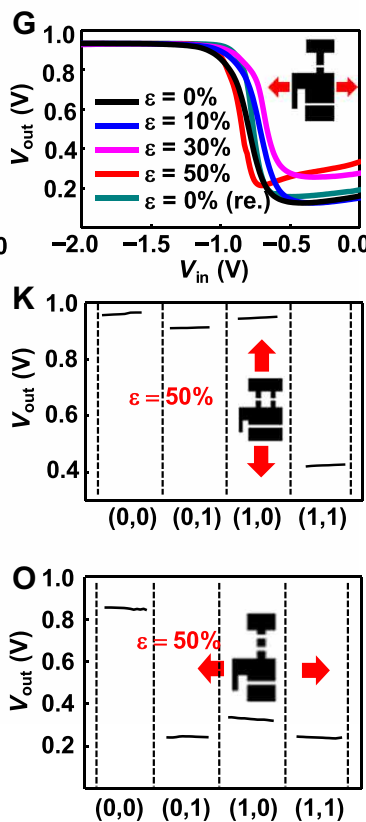

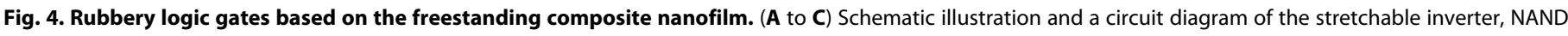

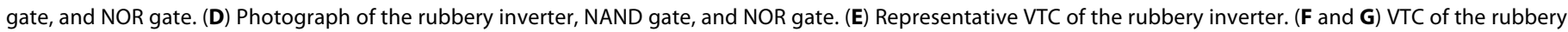

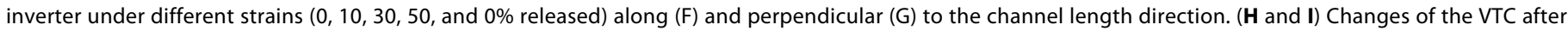

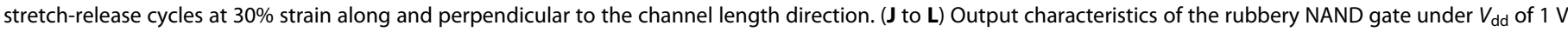

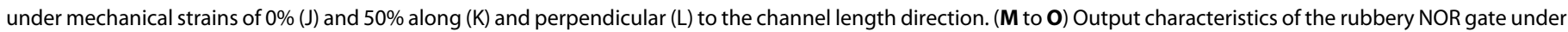
$V_{\mathrm{dd}}$ of $1 \mathrm{~V}$ under mechanical strains of $0 \%(\mathrm{M})$ and $50 \%$ along $(\mathrm{N})$ and perpendicular $(\mathrm{O})$ to the channel length direction. 
robustly even with applied mechanical strain, which paves the road toward highly complex integrated digital circuits.

\section{Multiplexed rubbery smart skin}

We developed an elastic smart skin based on the integration of the rubbery transistor active matrix and arrayed sensing node to illustrate one application of such rubbery-integrated electronics and functional systems. Specifically, the smart skin is structured with arrayed pressure sensing nodes made out of pressure-sensitive rubber and $5 \times 5$ rubbery transistors as an active matrix for multiplexing electrical readout, which is vital for robotics and skin prosthetics applications. The detailed smart skin fabrication process is described in Materials and Methods and is schematically illustrated in fig. S20. As shown in Fig. 5A, the fabricated fully rubbery smart skin conforms well on the back surface of a human hand. The left inset of Fig. 5A and fig. S21 shows the circuit diagram of an individual sensing node and the whole active matrix, respectively. The right inset of Fig. 5A depicts the optical image of the pixels. The exploded view of the active matrix smart sensory skin is schematically shown in Fig. 5B. A pressure-sensitive rubber sheet was serially connected to the drain of each transistor and supplied with $V_{\mathrm{dd}}$. The resistance of the pressure-sensitive rubber decreased from $\sim 200$ megohms to $8 \mathrm{ohms}$ when pressure was applied (fig. S22). The smart sensory skin shows excellent mechanical deformability as demonstrated by the representative mechanical deformations of twisting and poking (Fig. 5, C and D, respectively). The drain current change of a single sensing node with and without pressing is shown in Fig. 5E. The circuit diagram for characterizing the current is shown in fig. S23. The drain current markedly increases due to the decreased resistance of the pressure-sensitive rubber sheet under mechanical pressing. By serially connecting a shunt resistor ( 100 kilohms) to the source of the transistor (fig. S24), the voltage across the resistor can be measured to reflect the current and, thus, the pressed state on the skin. The dynamic output voltage response from a single sensing node based on a testing circuit is shown in Fig. 5F. The quantitative mapping of the output voltage of each multiplexed pixel was performed with a data acquisition system (DAQ, National Instruments). The detailed electrical interfaces with the DAQ are described in Materials and Methods. As shown in Fig. 5G, two fingers were placed on top of the sensor array. The corresponding voltage mapping is exhibited in Fig. $5 \mathrm{H}$. Even after stretching by $\sim 30 \%$, the skin can accurately resolve the pressed position (Fig. 5, I and J). These results illustrate the robust function of the rubbery smart skin.

\section{Medical robotic hand equipped with rubbery E-skin}

We further developed a medical robotic hand with a multifunctional E-skin to extract health information or provide medical stimulations through a direct interface with human body, which is an important
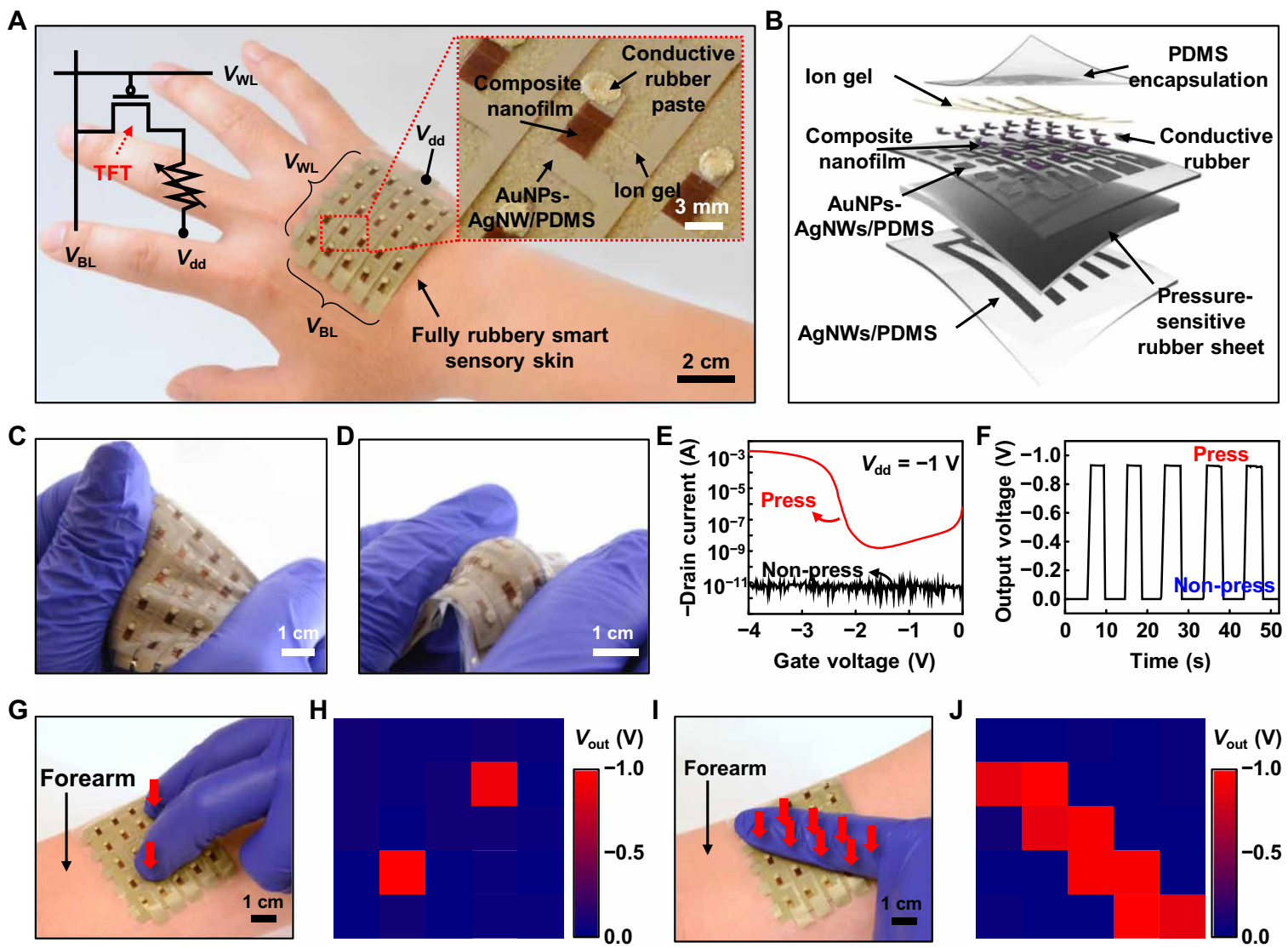

Fig. 5. Fully rubbery smart skin. (A) The rubbery smart skin adheres and conforms to the back of the human hand. The left inset shows the circuit diagram of the smart skin. (B) Schematic illustration of the smart skin. (C) Device under stretching and twisting deformations. (D) Device under poking deformation. (E) Transfer curve with/without pressing (circuit diagram for measurement is present in fig. S23). (F) Output voltage with/without pressing (circuit diagram for measurement is fig. S24). (G) Optical image of the smart skin pressed at two points. (H) Output voltage mapping for (G). (I) Optical image of the smart skin pressed in a line. (J) Output voltage mapping for (I). Photo credit: Ying-Shi Guan, University of Houston. 
and urgently needed device in medical fields. In particular, a deformable E-skin is indispensable for such medical devices to allow robotic hand motion, which is associated with large mechanical deformation. Specifically, the diagnostic E-skin comprised several components, including (i) transistor-based temperature sensors, (ii) a $2 \times 4$ array of multiplexed electrical stimulators, and (iii) a $3 \times 3$ array of multiplexed electrophysiological (EP) sensors. Specifically, the temperature sensors, electrical stimulators, and EP sensors were designed and positioned on the fingertips, the fingers, and the palm of a robotic hand, respectively. The detailed E-skin device fabrication processes are described in Materials and Methods. The photograph of the medical robotic hand with the E-skin is shown in Fig. 6A. The detailed structures and device dimensions are shown in figs. S25 and S26. The temperature sensor was characterized in the temperature range of 297 to $318 \mathrm{~K}\left(24^{\circ}\right.$ to $\left.45^{\circ} \mathrm{C}\right)$. The transistorbased temperature sensor works based on the variation of the off current of the device. Figure S27A shows the normalized channel resistance at a gate voltage of $-1 \mathrm{~V}$. The results are further plotted as $\ln (R)$ versus the reciprocal of the absolute temperature $(1 / T)$, which is well approximated by a linear curve, as shown in fig. S27B. These temperature sensing characteristics are similar to that of a typical negative temperature coefficient (NTC) thermistor. The calculated thermistor constant $(\beta)$ is 6827.22 . The temperature coefficient $(\alpha)$ is calculated to be from -7.73 to $-6.75 \% /{ }^{\circ} \mathrm{C}$ at the examined temperature range $\left(24^{\circ}\right.$ to $\left.45^{\circ} \mathrm{C}\right)$. To demonstrate its functionality, the robotic hand with E-skin touched a human forearm to measure the skin temperature, as shown in Fig. 6B. The measured temperature response for the room temperature and skin temperature is shown in Fig. 6C. The noise at certain locations is due to the disturbance in mechanical setup, movement of electrical wiring, and body dynamics, which might cause the contact issue of the measurement. The skin temperature is calculated to be $\sim 33^{\circ} \mathrm{C}$.

Low-frequency $(0.1$ to $1 \mathrm{~Hz})$ transcutaneous electrotherapies have been widely adopted for pain suppression, muscle reeducation, and
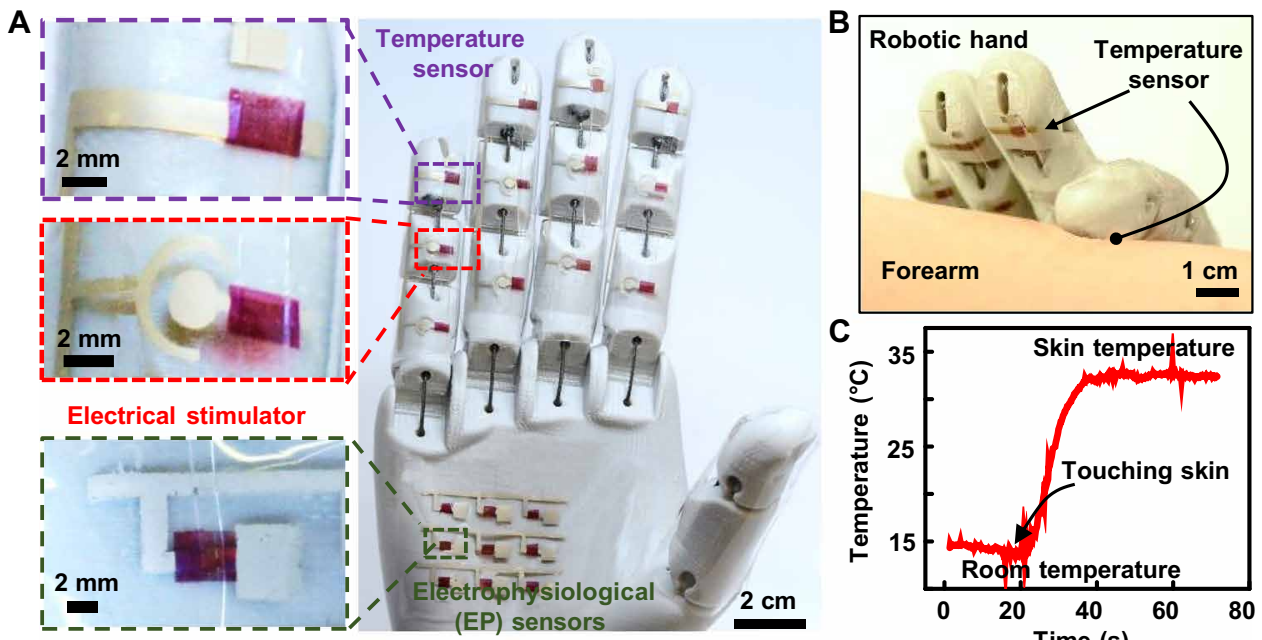

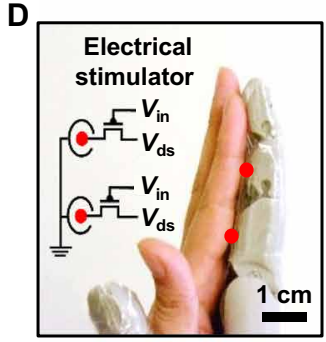

E

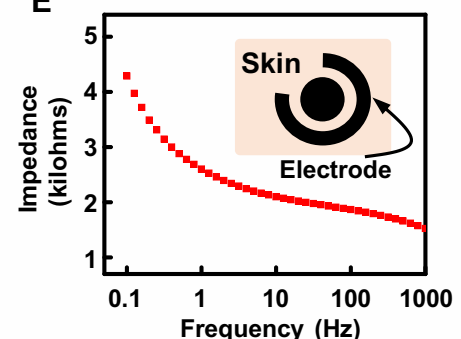

G
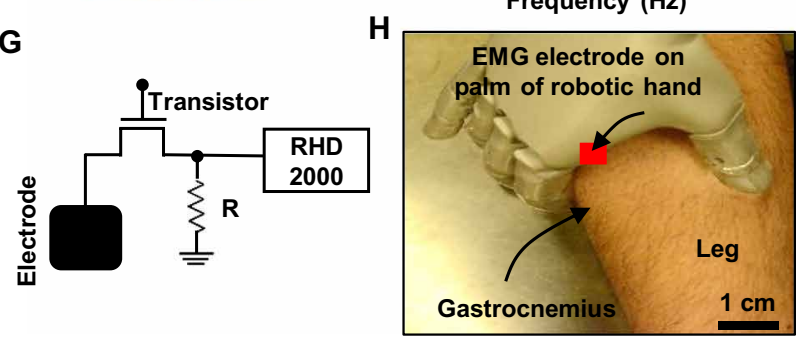

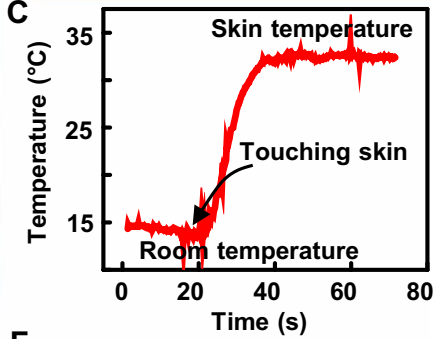

$F$
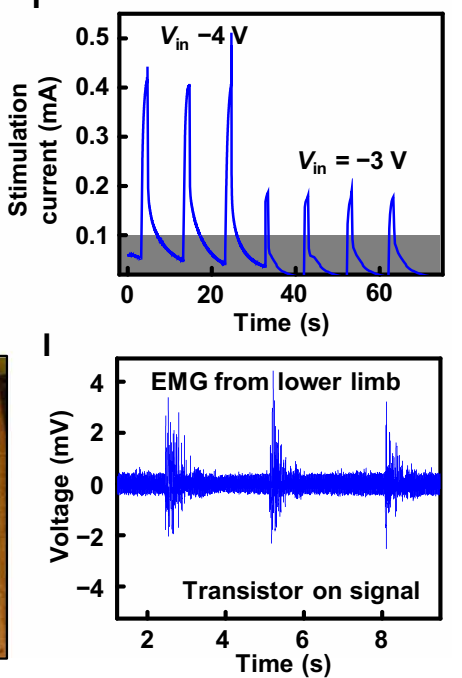

Fig. 6. A medical robotic hand equipped with rubbery E-skin. (A) Optical image of the medical robotic hand. (B) Robotic hand with E-skin touching a human forearm to measure the skin temperature. (C) Measured human skin temperature. (D) Optical image of the robotic hand with E-skin, contacting human muscles for electrical stimulation. (E) Human skin impedance measurement at different frequencies. Inset: Schematic diagram of the electrode. (F) Low-voltage electrical stimulation current peaks using the multiplexed electrical stimulators (signal from one pixel). (G) EMG measurement circuit using the rubbery transistor as a multiplexing switch. (H) Robotic hand with E-skin touching the lower limb for EMG measurement. (I) EMG signal from the posterior of the lower limb. Photo credit: Ying-Shi Guan, University of Houston. 
iontophoresis transdermal drug delivery (41). The optical image of the robotic hand with E-skin, contacting the human hand for electrical stimulation, is shown in Fig. 6D. We first measured the skin impedance coated with Ten 20 conductive gel (Weaver and Company), as it is important to know the impedance of the skin to trigger an electrical stimulation signal. The skin impedance measured at different frequencies is shown in Fig. 6E, which typically has an impedance value of several kilohms at low frequencies (42). The electrical circuit of the single pixel electrical stimulator is shown in fig. S28. The corresponding electrical stimulator circuit is shown in the inset of Fig. 6D. The stimulation current results with different applied $V_{\text {in }}$ are shown in Fig. 6F. The arrayed EP sensors on the robotic hand can potentially be used to monitor multiple EP signals when touching the human body. The electromyography (EMG) signal of a human subject was recorded with the Intan RHD2000. Figure 6G shows the EMG measurement circuit diagram using a fully rubbery transistor as a multiplexing switch. The EMG signal from rgw posterior section of the lower limb, particularly from the gastrocnemius muscle, was successfully acquired (Fig. 6, $\mathrm{H}$ and I), and the noise was filtered out using MATLAB. A clear and strong EMG signal could be observed. These results indicate the functionalities and capabilities of the medical hand with rubbery electronics-based multifunctional E-skin.

\section{DISCUSSION}

We demonstrated the facile and scalable manufacturing of rubbery semiconducting nanofilms based on the air/water interfacial assembly method. Such a manufacturing method is demonstrated to have high fidelity for large-area, high-quality P3HT semiconducting nanofilms. The molecular packing nature and the high carrier mobility of the assembled pristine and rubbery composite nanofilms further illustrate the advantage of such an assembly method. The unique P3HT- and SEBS-rich mesh-structured binary phases render the excellent mechanical stretchability in the rubbery semiconducting composite nanofilm. The demonstrated rubbery electronics, from the fundamental building block of transistors to logic gates to active matrix multiplexing electronics and to functional E-skins, show the potential of rubbery electronics and circuits, whose mechanical softness and stretchability stem from the intrinsic properties of the material used. This air/water interfacial assembly method could be potentially used for other electronic materials besides pristine and rubbery P3HT-based nanofilms, as a universal thinfilm material manufacturing platform. The development of rubbery semiconducting nanofilms and rubbery electronics paves the way for fully rubbery electronics and integrated systems and their applications in robotics and medical care, among others.

\section{MATERIALS AND METHODS Materials}

Anhydrous toluene (>99\%), SEBS, poly(vinylidene fluoride-cohexafluoropropylene) (PVDF-HFP; molecular weight, 400,000), 1-ethyl-3-methylimidazolium trifluoromethanesulfonate (EMIM-Otf; $>98 \%$ ), gold chloride trihydrate (>99.9\%), and anhydrous ammonia (28\%) were all purchased from Sigma-Aldrich and used as received. Regioregular P3HT (rrP3HT) was purchased from Sigma-Aldrich and washed and filtered to remove the low-molecular weight rrP3HT using cyclohexane. AgNW ( 99.5\%) solution (average diameter and length are $120 \mathrm{~nm}$ and $20 \mathrm{~mm}$, respectively) was from ACS
Material. PDMS rubber (Sylgard 184 silicone elastomer kit) was from Dow Corning. Pressure-sensitive rubber sheets (ZL45.1) and conductive rubber pastes (FL45) were from Zoflex.

\section{Fabrication of stretchable AuNPs-AgNWs/PDMS electrodes}

The fabrication of the stretchable AuNPs-AgNWs/PDMS electrodes follows the following steps. First, a shadow mask of Kapton film was manufactured using a programmable cutting machine (Silhouette Cameo). Second, the AgNW solution was drop-casted onto a cleaned glass slide with the shadow mask followed by curing at $60^{\circ} \mathrm{C}$ for $10 \mathrm{~min}$ and then at $200^{\circ} \mathrm{C}$ for $30 \mathrm{~min}$ on a hot plate. Third, a premixed PDMS solution [10:1 (w/w) prepolymer/curing agent] was spin-coated on the patterned AgNWs at $300 \mathrm{rpm}$ for $60 \mathrm{~s}$, followed by curing for 4 hours at $60^{\circ} \mathrm{C}$ to solidify. The PDMS was then peeled off from the glass slide to obtain the patterned AgNWs embedded in PDMS. Fourth, the AgNW/PDMS electrode was immersed in $0.5 \mathrm{mM} \mathrm{HAuCl}{ }_{4} \cdot \mathrm{H}_{2} \mathrm{O}$ aqueous solution for $2 \mathrm{~min}$ for the Ag-Au galvanic replacement to form AuNPs coated on AgNWs and dipped in $\mathrm{NH}_{4} \mathrm{OH}$ solution (28\%) for $1 \mathrm{~min}$ to dissolve $\mathrm{AgCl}$ layers that were formed on the NWs. Last, the fabrication of stretchable electrodes was accomplished by washing with deionized water and drying with a $\mathrm{N}_{2}$ gun. The schematic structure and the detailed geometries of the transistor are shown in fig. S29.

\section{Fabrication of the pristine P3HT nanofilm}

Ten milligrams of P3HT was dissolved in $1 \mathrm{ml}$ of toluene. Then, $10 \mu \mathrm{l}$ of solution was dropped onto the surface of water and the polymer solution was spread spontaneously and rapidly onto the water surface due to the Marangoni effect. After several minutes, the uniform pristine P3HT nanofilm formed over a large area. The obtained nanofilm can be transferred onto any substrates such as silicon, glass, and PDMS.

\section{Fabrication of the composite nanofilm}

SEBS and P3HT with different weight ratios were dissolved in $1 \mathrm{ml}$ of toluene. Then, $10 \mu \mathrm{l}$ of the blend polymer solution was dropped onto the surface of water to form the uniform composite nanofilm over a large area. The obtained composite nanofilm was used for further studies.

\section{Fabrication of the ion gel dielectric layer}

First, PVDF-HFP, EMIM-Otf, and acetone were mixed together with a weight ratio of $1: 4: 7$ at $70^{\circ} \mathrm{C}$. Then, the solution was casted onto a glass slide and cured for 12 hours at $70^{\circ} \mathrm{C}$ in a vacuum oven. The obtained ion gel layer can be conveniently cut into any shapes for use.

\section{Fabrication of the rubbery transistors, transistor array, and logic gates}

First, the freestanding composite nanofilm was transferred onto the patterned stretchable AuNPs-AgNWs/PDMS electrodes, prepared as previously reported (24), which served as the source and drain of the transistor. Then, the devices were naturally air-dried overnight. After that, the devices were dried at $60^{\circ} \mathrm{C}$ for $120 \mathrm{~min}$ in vacuum oven to totally remove the water. At last, the ion gel layer was laminated onto the composite nanofilm as the dielectric layer to complete the device fabrication. For the fabrication of the logic gates, all the procedures were the same as those of the rubbery transistors, except that the AuNPs-AgNWs/PDMS electrodes were patterned differently. 


\section{Fabrication of the active matrix for the smart skin}

To fabricate the fully rubbery smart sensory skin, the active matrix was fabricated using the procedures described for the fabrication of the transistor array. Next, via holes were formed to interconnect between the active matrix and a pressure-sensitive rubber sheet (ZL45.1, Zoflex). The conductive rubbery paste (FL45, Zoflex) was used to fill the via holes and was solidified at room temperature for 12 hours. The substantially strong adhesion between the solidified conductive rubber paste and PDMS does not allow delamination under a mechanical strain of $30 \%$. The active matrix and pressure-sensitive rubber sheet were encapsulated by a thin layer of PDMS (10:1 weight ratio of base and curing agent) by spin casting at $300 \mathrm{rpm}$ for $60 \mathrm{~s}$. Last, the smart sensory skin was laminated onto the patterned AgNWs/PDMS electrode to complete the device fabrication.

\section{Fabrication of the devices for the medical robotic hand}

The medical robotic hand comprises an array on the fingers and an active multiplexed array on the palm. The array of devices on each finger consists of a temperature sensor at the fingertip (distal phalanx) and electrical stimulators on the middle and proximal phalanxes. The electrical stimulators are multiplexed with thin-film transistors (TFTs) as switches. The dimensions of each device are shown in fig. S25. Similarly, a $3 \times 3$ array of EP electrodes was actively multiplexed at the palm of diagnostic robotic hand (fig. S26). The electrical stimulators and the EP electrodes comprise intrinsically stretchable AgNWs/PDMS electrodes and interconnections. The TFTs and circuits were fabricated as mentioned previously. The multiplexed diagnostic devices were then laminated around the 3D-printed tendon-operated robotic hand.

\section{Electrical characterization}

Electrical transport properties were measured under ambient conditions using a semiconductor analyzer (Keithley 4200-SCS) equipped with a probe station (H100, Signatone). A power supply (GPS-3303, GW Instek) was used to apply external voltage for the inverter, NAND, and NOR characterization. The electrical properties of a single sensing node were characterized with Keithley 4200SCS. We calculated mobility value on the basis of the specific capacitance of the ion gel of $9.8 \mu \mathrm{F} / \mathrm{cm}^{2}$ at $1 \mathrm{~Hz}$ using an LCR meter (Keysight, U1252B) and an oscilloscope (Agilent, DSO-X- 2004A), which agrees well with the values in literatures elsewhere (38). The output voltage mapping from the $5 \times 5$ active matrix-based fully rubbery smart skin was obtained from a DAQ and a custom LabView program. The voltage for the five-word lines and $V_{\mathrm{dd}}$ was applied by NI PXI-6723 (National Instruments) through an SCB-68 shielded input-output (I-O) connector block. The 5-bit lines were interfaced to NI PXI-6363 (National Instruments) through an SCB-68 shielded I-O connector block for readout of the output voltages. The temperature sensor was characterized using a hot plate to heat the device and then recording the electrical current with Keithley 4200 -SCS. The calibration was performed simultaneously with a digital infrared thermometer (Fluke 572-2). The EMG measurement was recorded with a DAQ (Intan RHD2000), where the output voltage was provided by a power supply (GPS-3303, GW Instek). The recorded EMG data were then filtered using MATLAB.

\section{Testing the devices on human hands}

The hands shown in Figs. 5 and 6 are those of Y.-S.G., who has given his consent to publish these images.

\section{SUPPLEMENTARY MATERIALS}

Supplementary material for this article is available at http://advances.sciencemag.org/cgi/ content/full/6/38/eabb3656/DC1

\section{REFERENCES AND NOTES}

1. J. E. Sosa-Hernández, A. M. Villalba-Rodríguez, K. D. Romero-Castillo, M. A. Aguilar-Aguila-Isaías, I. E. García-Reyes, A. Hernández-Antonio, I. Ahmed, A. Sharma, R. Parra-Saldívar, H. M. N. Iqbal, Organs-on-a-chip module: A review from the development and applications perspective. Micromachines 9, 536 (2018).

2. J. Byun, Y. Lee, J. Yoon, B. Lee, E. Oh, S. Chung, T. Lee, K.-J. Cho, J. Kim, Y. Hong, Electronic skins for soft, compact, reversible assembly of wirelessly activated fully soft robots. Sci. Robot. 3, eaas9020 (2018).

3. C. M. Boutry, M. Negre, M. Jorda, O. Vardoulis, A. Chortos, O. Khatib, Z. Bao, A hierarchically patterned, bioinspired e-skin able to detect the direction of applied pressure for robotics. Sci. Robot. 3, eaau6914 (2018).

4. J. Kim, A. S. Campbell, B. E.-F. de Ávila, J. Wang, Wearable biosensors for healthcare monitoring. Nat. Biotechnol. 37, 389-406 (2019).

5. K. Liu, Y. Jiang, Z. Bao, X. Yan, Skin-inspired electronics enabled by supramolecular polymeric materials. CCS Chemistry 1, 431-447 (2019).

6. R. Ma, S.-Y.Chou, Y. Xie, Q. Pei, Morphological/nanostructural control toward intrinsically stretchable organic electronics. Chem. Soc. Rev. 48, 1741-1786 (2019).

7. J. Rogers, Z. Bao, T.-W. Lee, Wearable bioelectronics: Opportunities for chemistry Acc. Chem. Res. 52, 521-522 (2019).

8. T. D. Y. Kozai, N. B. Langhals, P. R. Patel, X. Deng, H. Zhang, K. L. Smith, J. Lahann, N. A. Kotov, D. R. Kipke, Ultrasmall implantable composite microelectrodes with bioactive surfaces for chronic neural interfaces. Nat. Mater. 11, 1065-1073 (2012).

9. L. Xu, S. R. Gutbrod, A. P. Bonifas, Y. Su, M. S. Sulkin, N. Lu, H.-J. Chung, K.-I. Jang, Z. Liu, M. Ying, C. Lu, R. C. Webb, J.-S. Kim, J. I. Laughner, H. Cheng, Y. Liu, A. Ameen, J.-W. Jeong, G.-T. Kim, Y. Huang, I. R. Efimov, J. A. Rogers, 3D multifunctional integumentary membranes for spatiotemporal cardiac measurements and stimulation across the entire epicardium. Nat. Commun. 5, 3329 (2014).

10. S. I. Park, D. S. Brenner, G. Shin, C. D. Morgan, B. A. Copits, H. U. Chung, M. Y. Pullen, K. N. Noh, S. Davidson, S. J. Oh, J. Yoon, K.-I. Jang, V. K. Samineni, M. Norman, J. G. Grajales-Reyes, S. K. Vogt, S. S. Sundaram, K. M. Wilson, J. S. Ha, R. Xu, T. Pan, T.-i. Kim, Y. Huang, M. C. Montana, J. P. Golden, M. R. Bruchas, R. W. Gereau IV, J. A. Rogers, Soft, stretchable, fully implantable miniaturized optoelectronic systems for wireless optogenetics. Nat. Biotechnol. 33, 1280-1286 (2015).

11. H. Fang, K. J. Yu, C. Gloschat, Z. Yang, E. Song, C.-H. Chiang, J. Zhao, S. M. Won, S. Xu, M. Trumpis, Y. Zhong, S. W. Han, Y. Xue, D. Xu, S. W. Choi, G. Cauwenberghs, M. Kay, Y. Huang, J. Viventi, I. R. Efimov, J. A. Rogers, Capacitively coupled arrays of multiplexed flexible silicon transistors for long-term cardiac electrophysiology. Nat. Biomed. Eng. 1, 0038 (2017)

12. D.-Y. Khang, H. Jiang, Y. Huang, J. A. Rogers, A stretchable form of single-crystal silicon for high-performance electronics on rubber substrates. Science 311, 208-212 (2006).

13. Y. Sun, W. M. Choi, H. Jiang, Y. Y. Huang, J. A. Rogers, Controlled buckling of semiconductor nanoribbons for stretchable electronics. Nat. Nanotechnol. 1, 201-207 (2006).

14. A. Chortos, J. Lim, J. W. F. To, M. Vosgueritchian, T. J. Dusseault, T.-H. Kim, S. Hwang Z. Bao, Highly stretchable transistors using a microcracked organic semiconductor. Adv. Mater. 26, 4253-4259 (2014).

15. M. Shin, J. H. Song, G.-H. Lim, B. Lim, J.-J. Park, U. Jeong, Highly stretchable polymer transistors consisting entirely of stretchable device components. Adv. Mater. 26, 3706-3711 (2014).

16. J. Y. Oh, S. Rondeau-Gagné, Y.-C. Chiu, A. Chortos, F. Lissel, G.-J. N. Wang, B. C. Schroeder, T. Kurosawa, J. Lopez, T. Katsumata, J. Xu, C. Zhu, X. Gu, W.-G. Bae, Y. Kim, L. Jin, J. W. Chung, J. B.-H. Tok, Z. Bao, Intrinsically stretchable and healable semiconducting polymer for organic transistors. Nature 539, 411-415 (2016).

17. E. Song, B. Kang, H. H. Choi, D. H. Sin, H. Lee, W. H. Lee, K. Cho, Stretchable and transparent organic semiconducting thin film with conjugated polymer nanowires embedded in an elastomeric matrix. Adv. Electron. Mater. 2, 1500250 (2016).

18. G. Zhang, M. McBride, N. Persson, S. Lee, T. J. Dunn, M. F. Toney, Z. Yuan, Y.-H. Kwon, P.-H. Chu, B. Risteen, E. Reichmanis, Versatile interpenetrating polymer network approach to robust stretchable electronic devices. Chem. Mater. 29, 7645-7652 (2017).

19. Y.-S. Guan, H. Li, F. Ren, S. Ren, Kirigami-inspired conducting polymer thermoelectrics from electrostatic recognition driven assembly. ACS Nano 12, 7967-7973 (2018).

20. Y.-S. Guan, Z. Zhang, Y. Tang, J. Yin, S. Ren, Kirigami-inspired nanoconfined polymer conducting nanosheets with 2000\% stretchability. Adv. Mater. 30, 1706390 (2018).

21. K. Sim, Z. Rao, F. Ershad, C. Yu, Rubbery electronics fully made of stretchable elastomeric electronic materials. Adv. Mater. 32, 1902417 (2019).

22. C. Zhu, A. Chortos, Y. Wang, R. Pfattner, T. Lei, A. C. Hinckley, I. Pochorovski, X. Yan, J. W.-F. To, J. Y. Oh, J. B.-H. Tok, Z. Bao, B. Murmann, Stretchable temperature-sensing 
circuits with strain suppression based on carbon nanotube transistors. Nat. Electron. 1, 183-190 (2018).

23. M. Shin, J. Y. Oh, K.-E. Byun, Y.-J. Lee, B. Kim, H.-K. Baik, J.-J. Park, U. Jeong, Polythiophene nanofibril bundles surface-embedded in elastomer: A route to a highly stretchable active channel layer. Adv. Mater. 27, 1255-1261 (2015).

24. H.-J. Kim, K. Sim, A. Thukral, C. Yu, Rubbery electronics and sensors from intrinsically stretchable elastomeric composites of semiconductors and conductors. Sci. Adv. 3, e1701114 (2017).

25. J. Xu, S. Wang, G.-J. N. Wang, C. Zhu, S. Luo, L. Jin, X. Gu, S. Chen, V. R. Feig, J. W. F. To, S. Rondeau-Gagné, J. Park, B. C. Schroeder, C. Lu, J. Y. Oh, Y. Wang, Y.-H. Kim, H. Yan, R. Sinclair, D. Zhou, G. Xue, B. Murmann, C. Linder, W. Cai, J. B.-H. Tok, J. W. Chung, Z. Bao, Highly stretchable polymer semiconductor films through the nanoconfinement effect. Science 355, 59-64 (2017).

26. K. Sim, Z. Rao, H.-J. Kim, A. Thukral, H. Shim, C. Yu, Fully rubbery integrated electronics from high effective mobility intrinsically stretchable semiconductors. Sci. Adv. 5, eaav5749 (2019).

27. J. Xu, H.-C. Wu, C. Zhu, A. Ehrlich, L. Shaw, M. Nikolka, S. Wang, F. Molina-Lopez, X. Gu, S. Luo, D. Zhou, Y.-H. Kim, G.-J. N. Wang, K. Gu, V. R. Feig, S. Chen, Y. Kim, T. Katsumata, Y.-Q. Zheng, H. Yan, J. W. Chung, J. Lopez, B. Murmann, Z. Bao, Multi-scale ordering in highly stretchable polymer semiconducting films. Nat. Mater. 18, 594-601 (2019).

28. R. Tadmor, Marangoni flow revisited. J. Colloid Interface Sci. 332, 451-454 (2009).

29. A. Miniewicz, S. Bartkiewicz, H. Orlikowska, K. Dradrach, Marangoni effect visualized in two-dimensions Optical tweezers for gas bubbles. Sci. Rep. 6, 34787 (2016).

30. Y.-S. Guan, Y. Qin, Y. Sun, J. Chen, W. Xu, D. Zhu, Donor-acceptor co-assembled supramolecular nanofibers with high and well-balanced ambipolar charge transport properties under ambient conditions. Chem. Commun. 52, 4648-4651 (2016).

31. Y.-J. Kim, H.-T. Jung, C. W. Ahn, H.-J. Jeon, Simultaneously induced self-assembly of poly(3-hexylthiophene) ( $\mathrm{P} 3 \mathrm{HT})$ nanowires and thin-film fabrication via solution-floating method on a water substrate. Adv. Mater. Interfaces 4, 1700342 (2017).

32. Y. Yang, Z. Liu, J. Chen, Z. Cai, Z. Wang, W. Chen, G. Zhang, X. Zhang, L. Chi, D. Zhang, A facile approach to improve interchain packing order and charge mobilities by self-assembly of conjugated polymers on water. Adv. Sci. 5, 1801497 (2018).

33. S. Oh, S. Kang, M. H. M. Cativo, M. Yang, S.-H. Chung, J. Kim, J. Bouffard, S. Hong, S.-J. Park, Long-range order self-assembly of conjugated block copolymers at inclined air-liquid interfaces. ACS Appl. Mater. Interfaces 12, 5099-5105 (2020).

34. G. M. Whitesides, B. Grzybowski, Self-assembly at all scales. Science $\mathbf{2 9 5}$, 2418-2421 (2002).
35. S. Khodaparast, F. Boulogne, C. Poulard, H. A. Stone, Water-based peeling of thin hydrophobic films. Phys. Rev. Lett. 119, 154502 (2017).

36. G. Jo, J. Jung, M. Chang, Controlled self-assembly of conjugated polymers via a solvent vapor pre-treatment for use in organic field-effect transistors. Polymers 11, 332 (2019).

37. C. Gianino, Measurement of surface tension by the dripping from a needle. Phys. Educ. 41, 440-444 (2006).

38. K. H. Lee, M. S. Kang, S. Zhang, Y. Gu, T. P. Lodge, C. D. Frisbie, "Cut and stick" rubbery ion gels as high capacitance gate dielectrics. Adv. Mater. 24, 4457-4462 (2012).

39. F. S. Stinner, Y. Lai, D. B. Straus, B. T. Diroll, D. K. Kim, C. B. Murray, C. R. Kagan, Flexible, high-speed CdSe nanocrystal integrated circuits. Nano Lett. 15, 7155-7160 (2015).

40. T. Lei, L.-L. Shao, Y.-Q. Zheng, G. Pitner, G. Fang, C. Zhu, S. Li, R. Beausoleil, H.-S. P. Wong, T.-C. Huang, K.-T. Cheng, Z. Bao, Low-voltage high-performance flexible digital and analog circuits based on ultrahigh-purity semiconducting carbon nanotubes. Nat. Commun. 10, 2161 (2019).

41. M. A. Tooley, Medical physics and biomedical engineering. Physiol. Meas. 21, 549 (2000).

42. M. Ying, A. P. Bonifas, N. Lu, Y. Su, R. Li, H. Cheng, A. Ameen, Y. Huang, J. A. Rogers, Silicon nanomembranes for fingertip electronics. Nanotechnology 23, 344004 (2012).

\section{Acknowledgments}

Funding: C.Y. thanks the funding support by the NSF (CMMI-1554499), Office of Naval Research (N00014-18-1-2338) under the Young Investigator Program, NIH (R21EB026175). Author contributions: Y.-S.G. and C.Y. conceived and designed the experiment. Y.-S.G., A.T., K.S., X.W., Y.Z., and F.P. performed the experiment. S.Z. and J.X. performed the FEM simulation. Z.R. helped preparing the schematic diagram. Y.-S.G., P.W., and C.Y. analyzed experimental data. Y.-S.G., F.E., and C.Y. wrote the paper. Competing interests: The authors declare that they have no competing interests. Data and materials availability: All data needed to evaluate the conclusions in the paper are present in the paper and/or the Supplementary Materials. Additional data related to this paper may be requested from the authors.

Submitted 19 February 2020

Accepted 31 July 2020

Published 16 September 2020

10.1126/sciadv.abb3656

Citation: Y.-S. Guan, A. Thukral, S. Zhang, K. Sim, X. Wang, Y. Zhang, F. Ershad, Z. Rao, F. Pan, P. Wang, J. Xiao, C. Yu, Air/water interfacial assembled rubbery semiconducting nanofilm for fully rubbery integrated electronics. Sci. Adv. 6, eabb3656 (2020). 


\section{ScienceAdvances}

\section{Air/water interfacial assembled rubbery semiconducting nanofilm for fully rubbery integrated electronics}

Ying-Shi Guan, Anish Thukral, Shun Zhang, Kyoseung Sim, Xu Wang, Yongcao Zhang, Faheem Ershad, Zhoulyu Rao, Fengjiao Pan, Peng Wang, Jianliang Xiao and Cunjiang Yu

Sci Adv 6 (38), eabb3656.

DOI: $10.1126 /$ sciadv.abb3656

ARTICLE TOOLS

SUPPLEMENTARY

MATERIALS

REFERENCES

PERMISSIONS http://advances.sciencemag.org/content/6/38/eabb3656

http://advances.sciencemag.org/content/suppl/2020/09/14/6.38.eabb3656.DC1

This article cites 42 articles, 5 of which you can access for free http://advances.sciencemag.org/content/6/38/eabb3656\#BIBL

http://www.sciencemag.org/help/reprints-and-permissions

Science Advances (ISSN 2375-2548) is published by the American Association for the Advancement of Science, 1200 New York Avenue NW, Washington, DC 20005. The title Science Advances is a registered trademark of AAAS.

Copyright @ 2020 The Authors, some rights reserved; exclusive licensee American Association for the Advancement of Science. No claim to original U.S. Government Works. Distributed under a Creative Commons Attribution NonCommercial License 4.0 (CC BY-NC). 